\title{
NuSTAR Observation of a Minuscule Microflare in a Solar Active Region
}

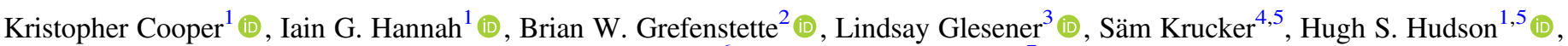 \\ Stephen M. White ${ }^{6}$ (D), and David M. Smith ${ }^{7}$ (D) \\ ${ }^{1}$ School of Physics \& Astronomy, University of Glasgow, Glasgow G12 8QQ, UK; k.cooper.2@ research.gla.ac.uk \\ ${ }^{2}$ Cahill Center for Astrophysics, 1216 East California Boulevard, California Institute of Technology, Pasadena, CA 91125, USA \\ ${ }^{3}$ School of Physics \& Astronomy, University of MinnesotaTwin Cities, Minneapolis, MN 55455, USA \\ ${ }^{4}$ University of Applied Sciences and Arts Northwestern Switzerland, 5210 Windisch, Switzerland \\ ${ }^{5}$ Space Sciences Laboratory University of California, Berkeley, CA 94720, USA \\ ${ }^{6}$ Air Force Research Laboratory, Space Vehicles Directorate, Kirtland AFB, NM 87123, USA \\ ${ }^{7}$ Santa Cruz Institute of Particle Physics and Department of Physics, University of California, Santa Cruz, CA 95064, USA \\ Received 2020 March 17; revised 2020 April 4; accepted 2020 April 7; published 2020 April 22
}

\begin{abstract}
We present X-ray imaging spectroscopy of one of the weakest active region (AR) microflares ever studied. The microflare occurred at 11:04 UT on 2018 September 9 and we studied it using the Nuclear Spectroscopic Telescope ARray (NuSTAR) and the Solar Dynamic Observatory's Atmospheric Imaging Assembly (SDO/AIA). The microflare is observed clearly in $2.5-7 \mathrm{keV}$ with NuSTAR and in Fe XVIII emission derived from the hotter component of the $94 \AA$ SDO/AIA channel. We estimate the event to be three orders of magnitude lower than a GOES A class microflare with an energy of $1.1 \times 10^{26} \mathrm{erg}$. It reaches temperatures of $6.7 \mathrm{MK}$ with an emission measure of $8.0 \times 10^{43} \mathrm{~cm}^{-3}$. Non-thermal emission is not detected but we instead determine upper limits to such emission. We present the lowest thermal energy estimate for an AR microflare in literature, which is at the lower limits of what is still considered an X-ray microflare.
\end{abstract}

Unified Astronomy Thesaurus concepts: Solar activity (1475); Solar corona (1483); Solar flares (1496); Solar x-ray flares (1816)

\section{Introduction}

Solar flares occur in active regions (ARs) and rapidly release stored magnetic energy into heating, mass flows, and particle acceleration in its vicinity (Benz 2017). The energy released can vary greatly, with smaller solar flares (called microflares) having energies about $10^{28}-10^{26}$ erg (Lin et al. 1984; Hannah et al. 2011). Microflares have been extensively studied in $\mathrm{X}$-rays, to determine their thermal and non-thermal properties and are observed to have GOES soft X-ray fluxes $<10^{-6} \mathrm{~W} \mathrm{~m}^{-2}$ and so are B, A, or sub-A class flares. Even smaller events (called nanoflares) were proposed by Parker (1988) as a unit of impulsive energy release to heat the whole corona, not just in ARs. These incredibly small events, with energies about $10^{24} \mathrm{erg}$, would need to be highly frequent with their frequency distribution having a power-law index $>2$ to dominate energetically over the larger flares (Crosby et al. 1993; Hudson 1991). The term nanoflare is sometimes used to describe an observed extreme-ultraviolet (EUV) brightening with energies about this scale.

X-ray emission from microflares has been extensively studied in the past with instruments such as the Reuven Ramaty High-Energy Solar Spectroscopic Imager (RHESSI; Lin et al. 2002). A comprehensive study of more than 25,000 microflaring events observed by RHESSI found that they shared many properties with their larger counterparts (Christe et al. 2008; Hannah et al. 2008). It was also noted that physical flare size did not seem to correlate with the magnitude of the microflare. To extend this work to even smaller flares requires improved sensitivity.

The Nuclear Spectroscopic Telescope ARray (NuSTAR) is an X-ray astrophysical telescope with the capability of observing the Sun above $2.5 \mathrm{keV}$ with unprecedented sensitivity (Harrison et al. 2013). NuSTAR consists of Wolter-I type optics on a $10 \mathrm{~m}$ mast that focus X-rays onto two focal plane modules (FPMA and FPMB), each with a field of view of $12^{\prime} \times 12^{\prime}$, made up of four pixelated CdZnTe detectors separated by chip gaps. NuSTAR detects individual counts, with a throughput of 400 counts $\mathrm{s}^{-1}$ module $^{-1}$. Even quiet or weakly flaring emission from the Sun can produce high count rates, resulting in significant deadtime and low effective exposure, thus most solar observations operate with a livetime fraction $\ll 1$ (Grefenstette et al. 2016). This can limit NuSTAR's sensitivity to the hottest material or weaker nonthermal energy during periods when livetime is small.

Since the first solar NuSTAR observations in 2014 (see Grefenstette et al. 2016; Hannah et al. 2016; Kuhar et al. 2017), solar activity has decreased allowing sub-A class microflares to be observed regularly within ARs (Glesener et al. 2017, 2020; Wright et al. 2017; Hannah et al. 2019) and small brightenings outside of ARs (Kuhar et al. 2018). ${ }^{8}$ The AR microflares observed by NuSTAR have been found to have thermal energy releases down to $10^{27} \mathrm{erg}$ with quiet Sun brightenings having energies down to $10^{26} \mathrm{erg}$. Non-thermal emission has rarely been observable in NuSTAR microflare analyses, with Glesener et al. (2020) reporting the first focusing optics imaging spectroscopy of non-thermal emission from an A5.7 class microflare. Limits on the non-thermal emission have been determined in other NuSTAR microflare analyses (Wright et al. 2017).

In this Letter, we present observations of a microflare from 2018 September 9 at $~ 11: 04$ UT (SOL2018-09-09T11) in AR AR12721. This event was observed with NuSTAR and also in EUV with the Solar Dynamic Observatory's Atmospheric Imaging Assembly (SDO/AIA; Lemen et al. 2012). In

\footnotetext{
8 Overview of all NuSTAR solar observations available at https://ianan. github.io/nsigh_all/.
} 
Section 2 the whole NuSTAR campaign, across 2018 September $9-10$, is briefly discussed. We then focus on the small microflare's time profiles and spatial properties (Section 2.1) followed by X-ray spectral analysis and GOES flare classification (Section 2.2). A thermal energy estimate is then calculated and compared to previously obtained values for other microflares in Section 2.3. An upper limit on the nonthermal emission of the microflare is also derived in Section 2.4. In Section 2.5, NuSTAR and SDO/AIA loci curves and emission measure distributions are calculated along with a comparison of the emission detected from both observatories.

\section{Weakest AR X-Ray Microflare}

NuSTAR performed six hour-long solar observations on 2018 September 9-10 with AR12721 (that emerged September 8) dominating the field of view. This campaign was related to a region targeted by the FOXSI-3 sounding rocket (Musset et al. 2019) on September 7, which was still in the NuSTAR field of view, but fainter and less active than AR12721. Numerous X-ray microflares produced by AR12721 were seen over the two-day observing window. In this Letter we focus on one of the smaller events; the other microflares are the subjects of a later paper.

\subsection{Time Profile and Imaging}

The microflare presented was initially discovered upon inspection of the NuSTAR lightcurve, shown in Figure 1, panel (a), calculated from the region shown in panel (b). The emission from the microflare becomes more pronounced above the background in the higher energy range of $4-7 \mathrm{keV}$ compared to $2.5-4 \mathrm{keV}$. A corresponding "bump" can be seen clearly in the SDO/AIA Fe XVIII proxy (Del Zanna 2013) but neither SDO/AIA $94 \AA$, nor the other SDO/AIA channels, displayed a clear feature. The $94 \AA$ images show a loop better than any other original SDO/AIA channel, but it is only in the Fe XVIII component that there is clear evidence of the microflare heating (Figure 1, panels (d) and (e)). Due to the position of the event on the NuSTAR focal plane, only the data obtained from FPMB can be used as the detector chip gap affects the FPMA data. It does, however, provide corroboratory evidence for the event as it also shows a clear microflare time profile.

It should be noted that, as expected of flaring behavior, it appears that the emission seen from the higher NuSTAR energy range, 4-7 keV, rises slightly before emission peaks in lower energies from NuSTAR $2.5-4 \mathrm{keV}$ and Fe XVIII. This could be due to hotter plasma earlier in the event or an initially accelerated electron distribution, a potential indication of nonthermal emission.

The NuSTAR pointing only requires a single correction over the entire time of the flare, found by aligning the NuSTAR image to SDO/AIA. We co-align the NuSTAR images with the Fe XVIII microflare emission map shown in panel (e). The shift in the NuSTAR pointing was obtained by cross-correlating the $\mathrm{X}$-ray and EUV images. Even after the spatial co-alignment, there still remains a conservative shift uncertainty of approximately $10^{\prime \prime}$. This is due to the lack of defined structure in the $\mathrm{X}$-ray image.

Contour maps of the shifted NuSTAR data on an Fe XVIII background during the pre-flare and microflare times are presented in Figure 2. The energy ranges are the same as those used in the lightcurves from Figure 1. Contours created from 2.5-4 keV (purple) and 4-7 keV (red) emission were deconvolved using a Lucy-Richardson method over 20 and 10 iterations, respectively (Richardson 1972). There is some 2.5-4 keV emission during the pre-flare time, which becomes brighter during the microflare and joined by the $4-7 \mathrm{keV}$ source at the same location.

To see the heating due to the microflare we subtract the preflare image from the microflare one-the resulting microflare excess is shown in Figure 2 (bottom panel). Here an elongated loop is more clearly visible in Fe XVIII and the $2.5-4 \mathrm{keV}$ source is similar to before. However, now the $4-7 \mathrm{keV}$ source is more elongated and the centroid is slightly shifted to the left of the $2.5-4 \mathrm{keV}$ source. This may not be a significant shift as it is within the spatial resolution of NuSTAR (Grefenstette et al. 2016). Although the time profile (Figure 1) and later the spectral fit results (Figure 3) show a definite but small event, the physical size of the microflaring loop $\left(\sim 20^{\prime \prime}\right.$ in length) is not uncommon from others observed in X-rays (Glesener et al. 2017; Hannah et al. 2008).

\subsection{NuSTAR Spectral Fitting}

In order to quantify thermal emission of the $\mathrm{AR}$ and microflare we fit the NuSTAR FPMB spectra (Figure 3). A circular region, centered on the brightest emission over the preflare and microflare times, with a radius of 26 " 5 , was used to produce both spectra.

The pre-flare spectrum (emission from 11:00:30 to 11:03:30 UT, Figure 3, left) is well fitted with a single APEC thermal model using XSPEC (Arnaud 1996). The fit gives a temperature of $3.2 \mathrm{MK}$ and an emission measure of $1.7 \times 10^{46} \mathrm{~cm}^{-3}$. These are typical values of quiescent/nonflaring ARs measured by NuSTAR (Glesener et al. 2017; Wright et al. 2017; Hannah et al. 2019). However, the livetime recorded throughout this event is significantly larger than those previously studied ( $\sim 15 \%$ compared to $1-5 \%)$, resulting in a better sensitivity, and hence constraint, on hotter material. This spectral fit was used as a fixed component during the microflare time (11:03:30 to 11:05:10 UT, Figure 3, right panel) with an additional APEC thermal component used to fit the microflare excess.

The microflare excess has a harder spectrum that dominates over the pre-flare emission $>4 \mathrm{keV}$, similar to what was found in Section 2.1. The event excess was fitted with a temperature of $6.7 \mathrm{MK}$ and an emission measure of $8.0 \times 10^{43} \mathrm{~cm}^{-3}$. The temperature is similar, or slightly hotter, to those found from other weak microflaring events, whereas the emission measure is an order of magnitude smaller (Glesener et al. 2017; Hannah et al. 2019).

The excess thermal fit does not change considerably when taking into account the uncertainty in the pre-flare model. It should be noted that because temperature and emission measure are inversely correlated, the highest/lowest temperature corresponds to the lowest/highest emission measure with asymmetric errors on each. We find that the temperature derived for the microflare excess through spectral fitting is consistent with the presence of emission in the SDO/AIA Fe XVIII channel. 

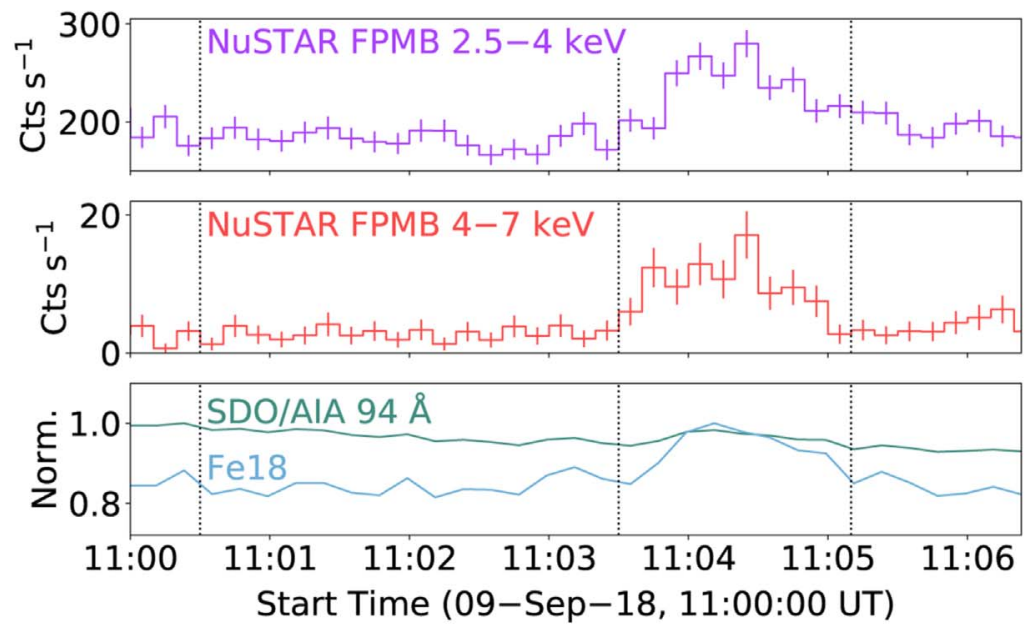

(a)

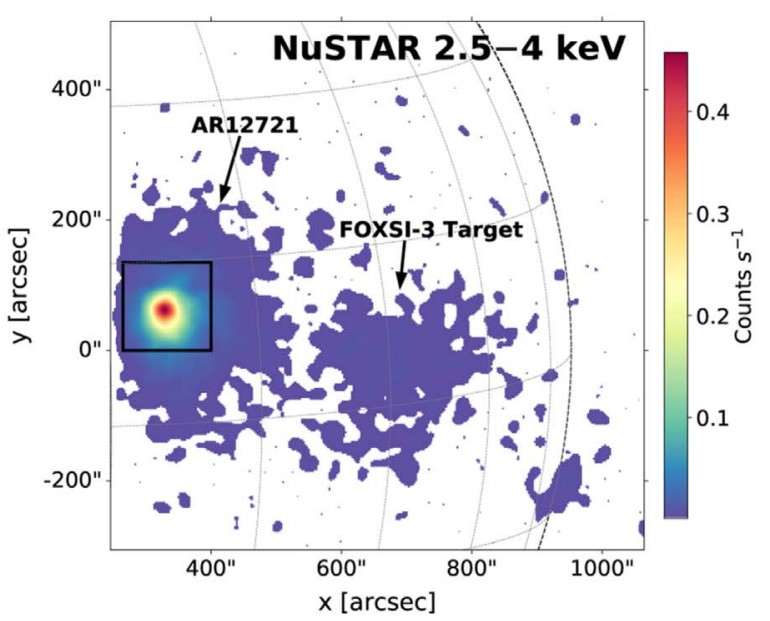

(b)

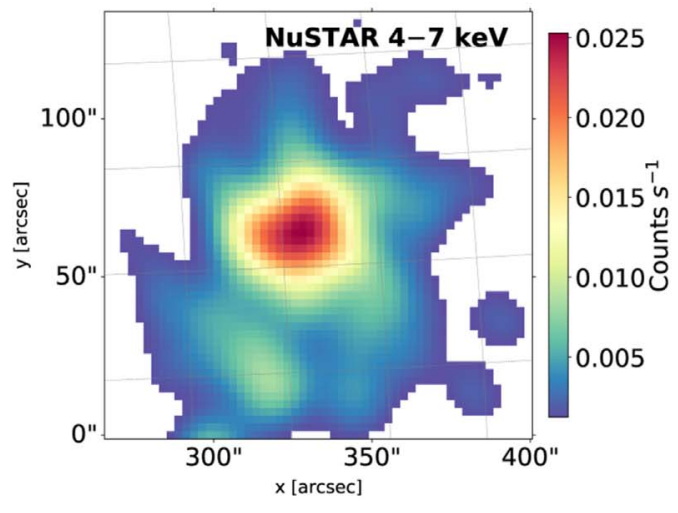

(c)

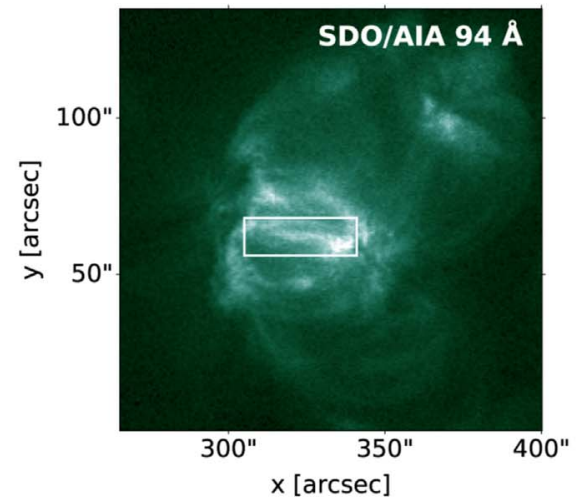

(d)

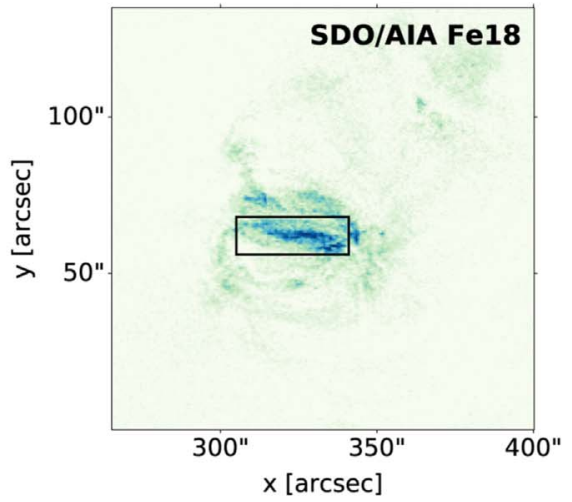

(e)

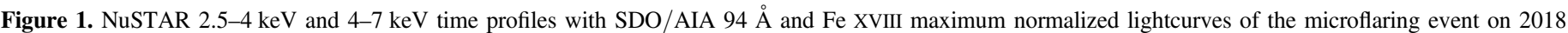

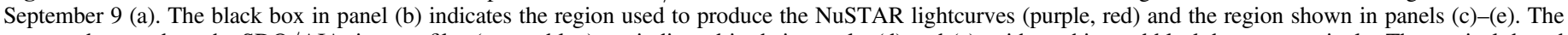

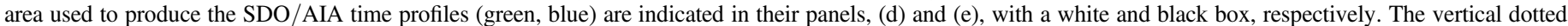

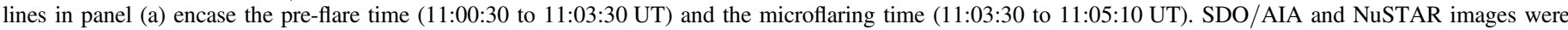

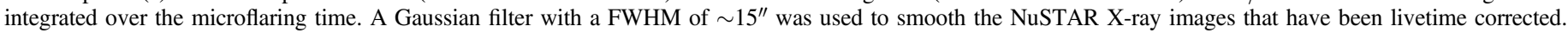

Using the goes_flux 49. pro $^{9}$ IDL routine in conjunction with the temperature and emission measure of the microflare excess, it is possible to obtain an estimated GOES classification for the event. We find a flux of $5 \times 10^{-11} \mathrm{~W} \mathrm{~m}^{-2}$, an equivalent GOES class of $\sim \mathrm{A} 0.005$.

\subsection{Thermal Energy}

From the temperature $(T)$ and emission measure (EM) of the microflare excess, with the addition of a volume estimate $(V)$ for the heated loop, the instantaneous thermal energy $\left(E_{\mathrm{th}}\right)$ can be calculated as

$$
E_{\mathrm{th}}=3 N_{e} k_{\mathrm{B}} T=3(V \times \mathrm{EM})^{\frac{1}{2}} k_{\mathrm{B}} T[\mathrm{erg}],
$$

where $N_{e}$ is the total number of thermal electrons and $k_{\mathrm{B}}$ is Boltzmann's constant (Hannah et al. 2008). The temperature and emission measure are taken from the microflare excess, given in Figure 3 (right panel). The volume of the loop can be estimated from the EUV SDO/AIA Fe XVIII image (Figure 2, bottom panel).

\footnotetext{
9 https://hesperia.gsfc.nasa.gov/ssw/gen/idl/synoptic/goes/goes_ flux 49.pro
}

The microflaring loop appears to be $22^{\prime \prime}$ by $2^{\prime \prime}$ (approximately $1.6 \times 10^{9}$ by $1.3 \times 10^{8} \mathrm{~cm}$ ). Taking the heated loop as a half-torus, this gives a volume of $3.2 \times 10^{25} \mathrm{~cm}^{3}$. Thus, using Equation (1) with a temperature of 6.7 MK and emission measure $8.0 \times 10^{43} \mathrm{~cm}^{-3}$, we find a thermal energy of $1.4_{-0.2}^{+0.3} \times$ $10^{26} \mathrm{erg}$. The volume estimated here is undoubtedly an upper limit as it could be contested that the region in EUV is smaller still as most of the emission appears to be focused at the right of the loop with surrounding fainter emission. In addition, this volume estimate does not consider a loop-filling factor, making the thermal energy estimate an upper limit. This thermal energy value is lower than the previous smallest observed NuSTAR microflare (Hannah et al. 2019), which was cooler but with a higher emission measure and had a GOES class of A0.02. EUV observations of magnetically braided loops observed heating with thermal energy of about $10^{26} \mathrm{erg}$ (Cirtain et al. 2013); however, this was for material up to $4 \mathrm{MK}$.

\subsection{Non-thermal Limits}

Following the approach in Wright et al. (2017), it is possible to obtain upper limits on any non-thermal emission produced by the microflare from NuSTAR's spectral response. This is 

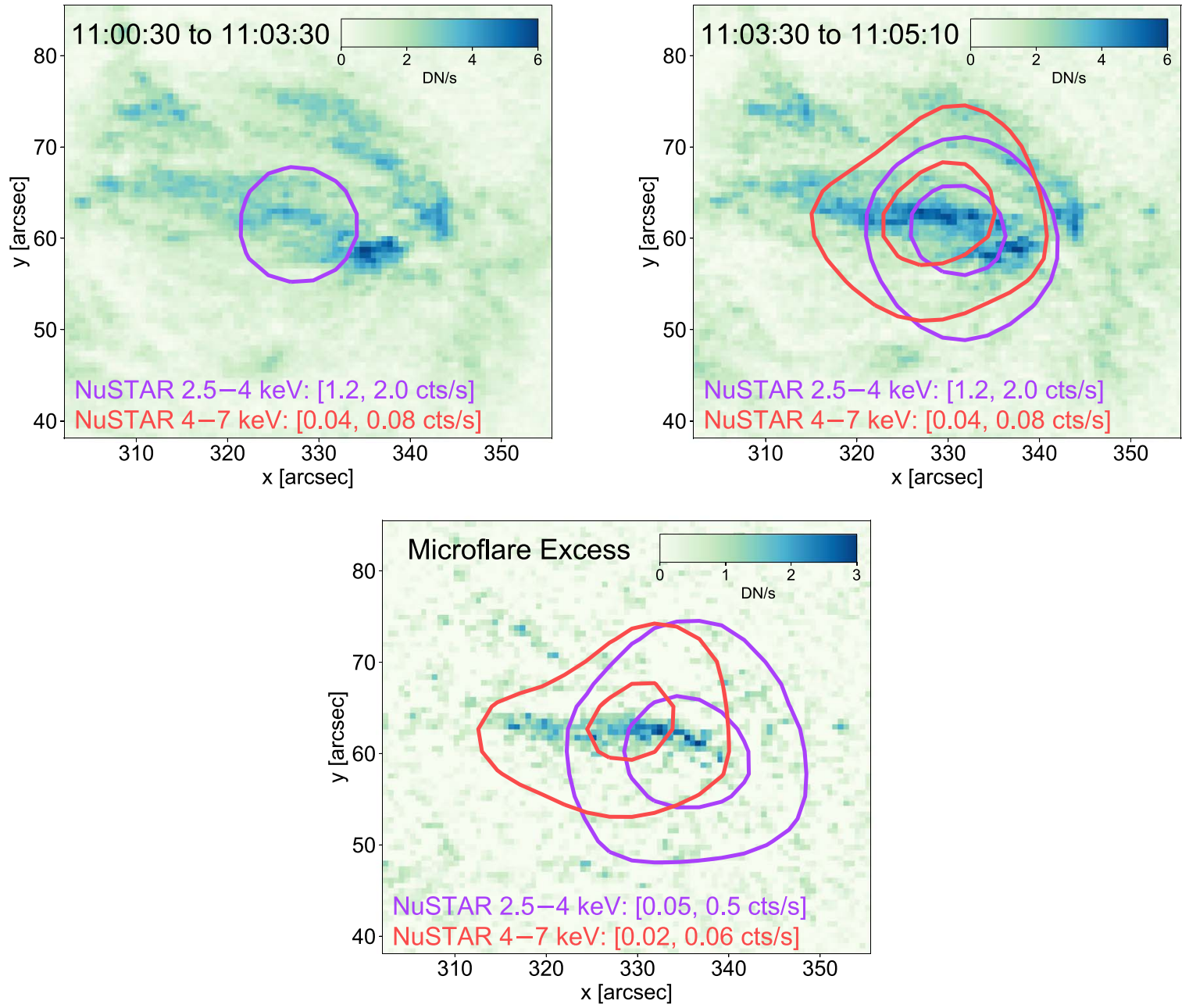

Figure 2. SDO/AIA Fe XVIII map with 2.5-4 keV and 4-7 keV NuSTAR absolute contour levels for the pre-flare time (top-left panel) and the microflaring time (topright panel). The bottom panel shows the the pre-flare subtracted map, i.e., the microflare excess.

done by adding a thick target model (f_thick $2 \cdot$ pro $^{10}$ ) to a simulated spectrum obtained from the total microflare thermal model. This non-thermal model depends on the power-law index, the low-energy cut-off, and the electron flux of the microflare accelerated electrons. The non-thermal power was calculated throughout this parameter space, where the thick target model gave fewer than four counts at energies greater than $7 \mathrm{keV}$ - consistent with a null detection to $2 \sigma$ (Gehrels 1986) - and that the introduced non-thermal counts were within Poissonian uncertainty at energies $\lesssim 7 \mathrm{keV}$. We find that the upper non-thermal limits produced are consistent with the required heating over the microflare time $\left(\sim 10^{24} \mathrm{erg} \mathrm{s}^{-1}\right)$ but only with low-energy cut-offs down to $\sim 6 \mathrm{keV}$ with a powerlaw index $\gtrsim 6$.

The upper limit values calculated that satisfy this microflare heating are lower than the upper limits in Wright et al. (2017). This is expected as the microflare discussed here is less energetic. The largest upper limit obtained from this analysis $\left(\sim 10^{25} \mathrm{erg} \mathrm{s}^{-1}\right)$ is only just comparable to the smallest nonthermal power in similar sized microflares (Hannah et al. 2008, Table 1). The power-law index and cut-off energy are consistent with the values obtained in Glesener et al. (2020). Only the electron flux is different $\left(\sim 10^{3}\right.$ larger $)$, which could be

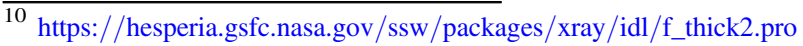

expected as the peak emission is also orders of magnitude larger than the flare discussed here. However, the values obtained are not consistent with the events presented in Testa et al. (2014), who investigated coronal loop footpoint brightenings in ultraviolet (UV) and a nanoflare heating model. Their model required that an electron distribution with a higher lowenergy cut-off $(\sim 10 \mathrm{keV})$ to match their observations compared to the microflare presented.

\subsection{Multi-thermal Microflare Analysis}

Figure 4 shows the EM loci curves (flux divided by temperature response) from SDO/AIA and NuSTAR plotted with the temperatures and emission measures obtained from Figure 3 and their $90 \%$ confidence region (hatched regions). During the pre-flare time (Figure 4, left), the Fe XVIII and NuSTAR loci curves almost intersect at the temperature and emission measure from the spectral fit. This indicates that similar emission is observed by NuSTAR and Fe XVIII at the pre-flare stage over the selected region (Hannah et al. 2019). The microflare time has the additional heated plasma from the flaring process (see Figures 3 and 4, right) which as expected, results in Fe XVIII and NuSTAR not intersecting at the same point, nor agreeing with the spectral fit value.

To determine the multi-thermal properties we calculate the emission measure distribution (EMD; the line-of-sight 

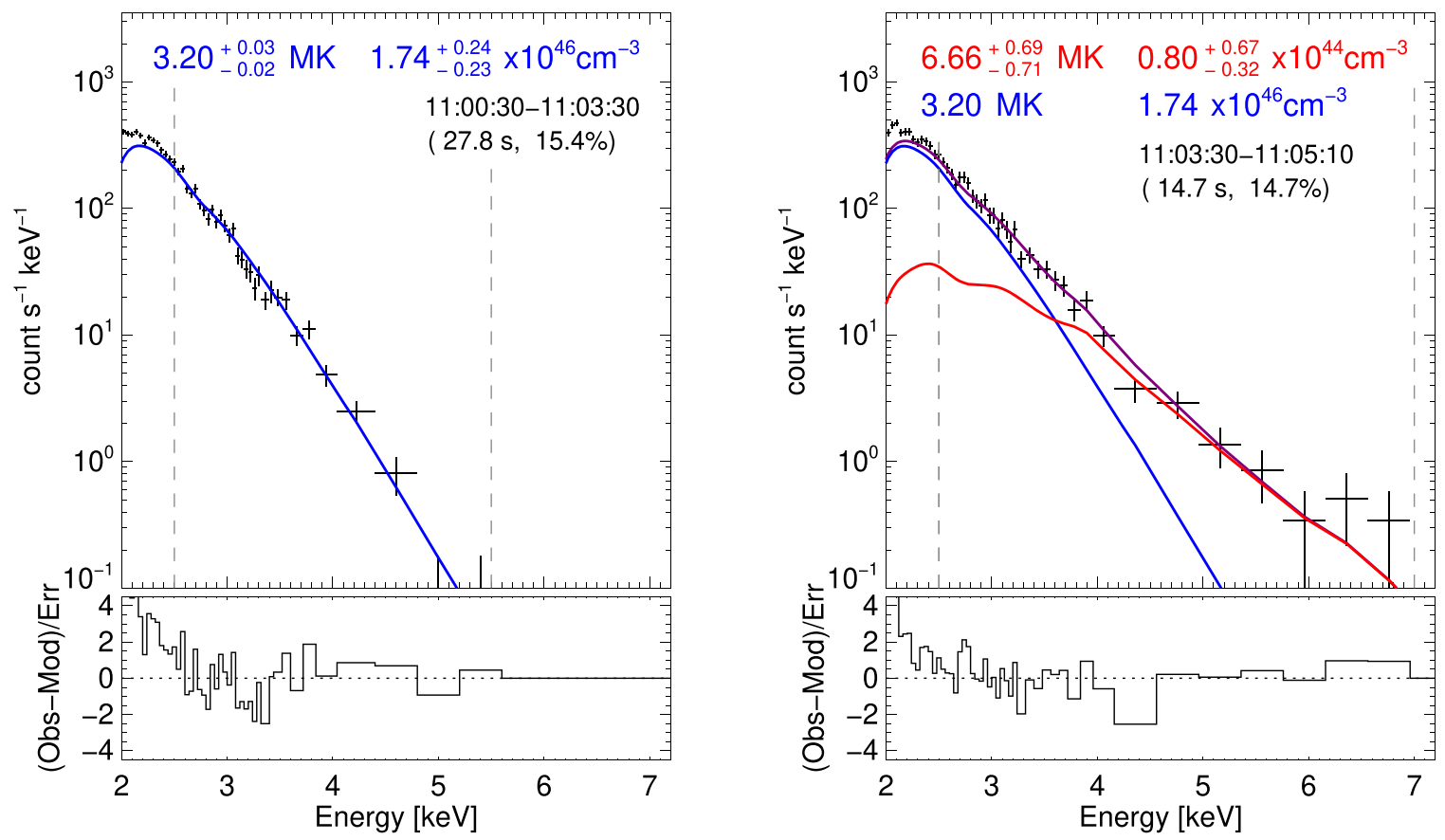

Figure 3. Thermal model fits, using XSPEC, of NuSTAR emission during the pre-flare time (left panel) and microflare time (right panel). The pre-flare spectrum is fitted with one thermal model (blue), which is then used as a fixed component for the microflare spectrum fit along with an additional thermal model (red). Both models in the microflare spectrum combine to give the overall model (purple). The temperatures, emission measures, and times ranges are shown for the spectra with their effective exposures and livetimes in brackets. The quoted errors denote a $90 \%$ confidence range with the fit over the energy range indicate by the vertical dashed lines.
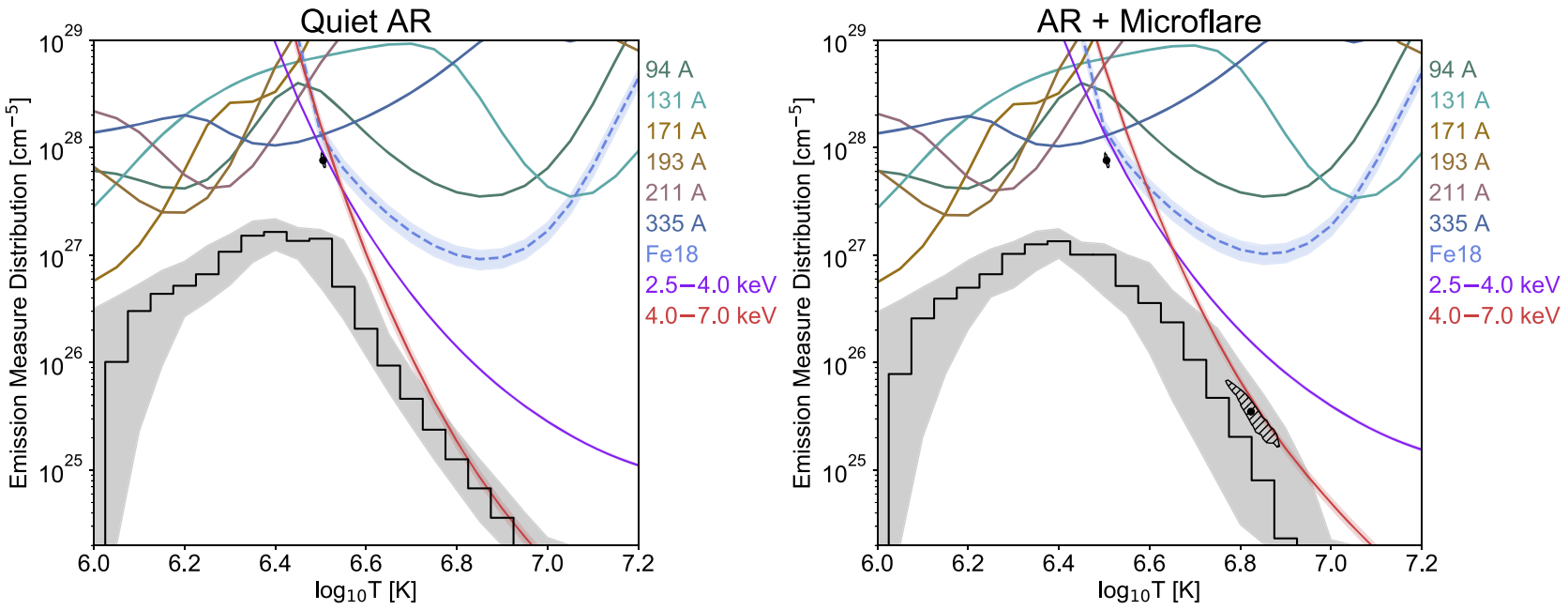

Figure 4. NuSTAR and SDO/AIA loci curve plots with the calculated emission measure distributions (black) for the pre-flare time (left panel) and microflare time (right panel). The shaded areas denote the uncertainty range for the NuSTAR loci curves (purple and red), the Fe XVIII curve (dashed, blue), and the emission measure distribution (gray). The region used to calculate the SDO/AIA and NuSTAR instrument loci curves was the boxed region shown in Figure 1, panels (d) and (e). The spectral fit values for both times are indicated with their hatched $90 \%$ confidence regions.

differential emission measure multiplied by the temperature bin width, in units of $\mathrm{cm}^{-5}$ ) using the regularized inversion approach of Hannah \& Kontar (2013). Both AIA and NuSTAR data were used and the resulting EMD curves, and uncertainty regions, are shown in Figure 4.

We find that, in Figure 4, the calculated EMDs are consistent with the values obtained from the spectral fits and the loci curve upper boundaries. The EMD indicates a sharp edge at the quiescent AR/pre-flare spectral fitting values (Figure 4, left panel) and a smoother drop in hotter material during the microflaring time (right panel). As the microflare heats the plasma an excess of material appears at temperatures where the "tail" of the pre-flare plasma falls off quickly. This behavior is similar to what has been found for significantly larger microflares, also observed in EUV and X-rays (Athiray et al. 2020). The pre-flare time EMD in Figure 4 (left panel) again shows the importance of higher-energy X-ray spectroscopy when trying to robustly determine the presence of hot material in non-flaring ARs, as highlighted in previous studies (Reale et al. 2009; Schmelz et al. 2009; Ishikawa et al. 2017).

By subtracting the pre-flare emission from the microflareisolating the microflare heated plasma-the loci curves show more consistent behavior with the spectral fit excess parameters 

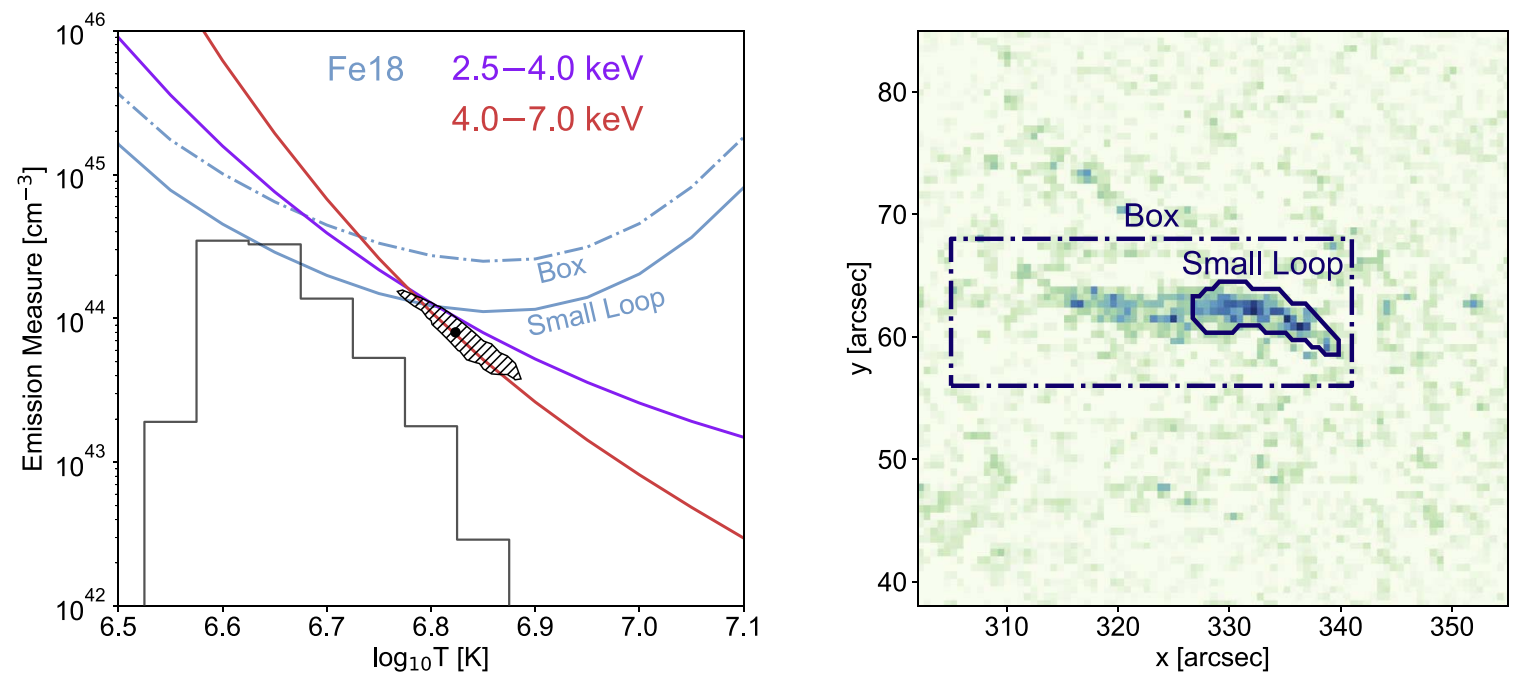

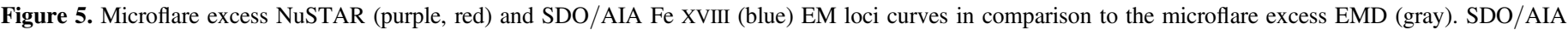

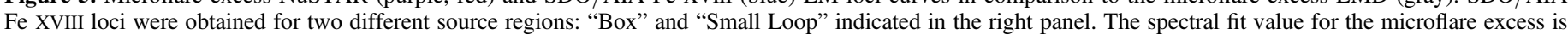
shown in the loci plot with a black dot and $90 \%$ confidence region where errors on all other quantities are omitted due to their large magnitude.

$\left(6.7 \mathrm{MK}\right.$ and $\left.8.0 \times 10^{43} \mathrm{~cm}^{-3}\right)$, again, indicating similar emission seen by both observatories (Figure 5, left panel).

Figure 5 (left panel) displays further evidence that the microflare emission is from the right-hand side of the region assumed in Section 2.1. The Fe XVIII (blue, solid) loci curve from the "Small Loop" area is far more consistent with the NuSTAR loci curves and spectral fit value compared to the larger "Box" region loci curve (blue, dashed-dotted). This indicates that during the microflare time the excess emission is observed from this "Small Loop" region and that the pre-flare emission was from a larger fraction of the AR.

Further support for this is seen when we compare the observed SDO/AIA Fe XVIII flux from these regions to the synthetic AIA flux, calculated from the NuSTAR thermal parameters folded through the AIA response. Using the AIA flux from the "Box" we find that NuSTAR appears to detect $\sim 62 \%$ of the emission observed by SDO/AIA Fe XVIII (synthetic flux: $1.20_{-0.09}^{+0.11} \mathrm{DN} \mathrm{s}^{-1} \mathrm{pixel}^{-1}$, observed flux: $1.95 \pm 0.06 \mathrm{DN} \mathrm{s}^{-1}$ pixel $^{-1}$ ) from the quiescent AR. However, only $\sim 30 \%$ of the microflare excess is observed by NuSTAR $\left(0.07_{-0.04}^{+0.06} \mathrm{DN} \mathrm{s}^{-1}\right.$ pixel $\left.^{-1}\right)$ compared to Fe XVIII $\left(0.23 \pm 0.09 \mathrm{DN} \mathrm{s}^{-1}\right.$ pixel $\left.^{-1}\right)$, whereas the synthetic flux obtained for the microflare excess in the "Small Loop" region $\left(1.14_{-0.57}^{+1.03} \mathrm{DN} \mathrm{s}^{-1}\right.$ pixel $\left.^{-1}\right)$ is $\sim 69 \%$ of the observed flux $\left(1.66 \pm 0.16 \mathrm{DN} \mathrm{s}^{-1}\right.$ pixel $\left.^{-1}\right)$. The smaller region is more consistent for the microflare excess with the temperature calculated and the Fe XVIII response. Thus, it is determined that the "Small Loop" region shown in Figure 5 is the true microflaring loop. A volume of $1.9 \times 10^{25} \mathrm{~cm}^{3}$ and energy of $1.1_{-0.2}^{+0.2} \times 10^{26} \mathrm{erg}$ is then recalculated for this smaller loop, lowering the already small upper limit given to the instantaneous energy release of this microflare.

\section{Summary and Conclusions}

Using NuSTAR, in conjunction with SDO/AIA, we have identified the smallest thermal energy X-ray microflare yet detected within an AR. A typical quiescent AR/pre-flare temperature and emission measure $\left(\sim 3 \mathrm{MK}\right.$ and $\sim 10^{46} \mathrm{~cm}^{-3}$, respectively) was obtained when fitting a thermal model to the spectrum with the microflare excess reaching temperatures up to $6.7 \mathrm{MK}$ and an emission measure of $8.0 \times 10^{43} \mathrm{~cm}^{-3}$. This is hotter and has a lower emission measure than most of the previously studied NuSTAR microflares (Hannah et al. 2016, 2019; Glesener et al. 2017; Wright et al. 2017).

The microflare is estimated to have a thermal energy release of $1.1_{-0.2}^{+0.2} \times 10^{26} \mathrm{erg}$. This is the not the most spatially compact microflare, but it is the smallest thermal energy release from an $\mathrm{X}$-ray microflare observed in an AR. This thermal energy is comparable to the small brightenings seen in high-resolution EUV observations of magnetically braided loops (Cirtain et al. 2013). This shows that with NuSTAR we are starting to detect the X-ray emission from the myriad of small brightenings seen in EUV, and are approaching events closer to nanoflare than microflare energies.

No non-thermal emission was detected; however, some electron acceleration could have occurred throughout the evolution of the microflare. Support for this comes in the form of the higher energy time profile (NuSTAR 4-7 keV) rising earlier than the lower energy profiles (NuSTAR 2.5-4 keV and SDO/AIA Fe XVIII). We found non-thermal upper limits that were consistent with not producing detectable emission, yet still capable of matching the heating rate in this microflare.

This tiny microflare was very evident in the X-ray data but harder to find in the EUV emission, highlighting the need for sensitive X-ray telescopes to study flares. It may be easier, however, to find more events of this scale within ARs, using this one as an example. This would further the investigation into how the flare frequency distribution of smaller flares compare to that of larger ones (Crosby et al. 1993; Hudson 1991; Hannah et al. 2011).

Throughout the six $\sim 1 \mathrm{~h}$ NuSTAR observations on 2018 September 9-10 there was a multitude of microflares from AR12721. The statistics of these events will be the subject of another paper, furthering our understanding of the range of small flares possible.

This Letter made use of data from the NuSTAR mission, a project led by the California Institute of Technology, managed by the Jet Propulsion Laboratory, funded by the National Aeronautics and Space Administration. These observations were supported through the NuSTAR Guest Observer program (NASA grant 80NSSC18K1744). This research has made use 
of SunPy v1.0.6, an open-source and free communitydeveloped solar data analysis Python package (SunPy Community et al. 2015). This research also made use of HEASoft (a unified release of FTOOLS and XANADU software packages) and NuSTAR Data Analysis Software (NuSTARDAS). This Letter made use of the SolarSoft IDL distribution (SSW) from the IDL Astronomy Library.

K.C. is supported by a Royal Society Research Fellows Enhancement Award and I.G.H is supported by a Royal Society University Fellowship.

\section{ORCID iDs}

Kristopher Cooper (ib https://orcid.org/0000-0001-8589-3378

Iain G. Hannah (DD https://orcid.org/0000-0003-1193-8603

Brian W. Grefenstette (iD https://orcid.org/0000-0002-

1984-2932

Lindsay Glesener (i) https://orcid.org/0000-0001-7092-2703

Hugh S. Hudson (iD https://orcid.org/0000-0001-5685-1283

Stephen M. White (i) https://orcid.org/0000-0002-8574-8629

David M. Smith (iD https://orcid.org/0000-0002-0542-5759

\section{References}

Arnaud, K. A. 1996, in ASP Conf. Ser. 101, Astronomical Data Analysis Software and Systems V, ed. G. H. Jacoby \& J. Barnes (San Francisco, CA: ASP), 17

Athiray, P. S., Vievering, J., Glesener, L., et al. 2020, ApJ, 891, 78

Benz, A. O. 2017, LRSP, 14, 2
Christe, S., Hannah, I. G., Krucker, S., McTiernan, J., \& Lin, R. P. 2008, ApJ, 677, 1385

Cirtain, J. W., Golub, L., Winebarger, A. R., et al. 2013, Natur, 493, 501

Crosby, N. B., Aschwanden, M. J., \& Dennis, B. R. 1993, SoPh, 143, 275

Del Zanna, G. 2013, A\&A, 558, A73

Gehrels, N. 1986, ApJ, 303, 336

Glesener, L., Krucker, S., Duncan, J., et al. 2020, ApJL, 891, L34

Glesener, L., Krucker, S., Hannah, I. G., et al. 2017, ApJ, 845, 122

Grefenstette, B. W., Glesener, L., Krucker, S., et al. 2016, ApJ, 826, 20

Hannah, I. G., Christe, S., Krucker, S., et al. 2008, ApJ, 677, 704

Hannah, I. G., Grefenstette, B. W., Smith, D. M., et al. 2016, ApJL, 820, L14

Hannah, I. G., Hudson, H. S., Battaglia, M., et al. 2011, SSRv, 159, 263

Hannah, I. G., Kleint, L., Krucker, S., et al. 2019, ApJ, 881, 109

Hannah, I. G., \& Kontar, E. P. 2013, A\&A, 553, A10

Harrison, F. A., Craig, W. W., Christensen, F. E., et al. 2013, ApJ, 770, 103

Hudson, H. 1991, SoPh, 133, 357

Ishikawa, S.-n., Glesener, L., Krucker, S., et al. 2017, NatAs, 1, 771

Kuhar, M., Krucker, S., Glesener, L., et al. 2018, ApJL, 856, L32

Kuhar, M., Krucker, S., Hannah, I. G., et al. 2017, ApJ, 835, 6

Lemen, J. R., Title, A. M., Akin, D. J., et al. 2012, SoPh, 275, 17

Lin, R. P., Dennis, B. R., Hurford, G. J., et al. 2002, SoPh, 210, 3

Lin, R. P., Schwartz, R. A., Kane, S. R., Pelling, R. M., \& Hurley, K. C. 1984, ApJ, 283, 421

Musset, S., Buitrago-Casas, J. C., Glesener, L., et al. 2019, Proc. SPIE, 11118 , 1111812

Parker, E. N. 1988, ApJ, 330, 474

Reale, F., McTiernan, J. M., \& Testa, P. 2009, ApJL, 704, L58

Richardson, W. H. 1972, JOSA, 62, 55

Schmelz, J. T., Kashyap, V. L., Saar, S. H., et al. 2009, ApJ, 704, 863

SunPy Community, T., Mumford, S. J., Christe, S., et al. 2015, CS\&D, 8, 014009

Testa, P., Pontieu, B. D., Allred, J., et al. 2014, Sci, 346, 26

Wright, P. J., Hannah, I. G., Grefenstette, B. W., et al. 2017, ApJ, 844, 132 